\title{
网络社区交流中距离的作用 以新浪微博为例
}

\author{
王 波 ${ }^{1}$, 甄 峰 $2,3^{*}$ \\ (1. 香港大学地理系, 香港 $999077 ; 2$. 南京大学建筑与城市规划学院, 南京 210093; \\ 3. 南京大学人文地理研究中心, 南京 210093)
}

\begin{abstract}
摘 要: 信息通信技术的快速发展丰富了人们的交流方式,也逐步影响人们的社交网络。本文通过分析新浪微博用 户在网络社区中与本地人/外地人、熟人/陌生人的交流差异,探讨空间距离与社会距离在网络社会空间中的作用。 在问卷调研中, 结合新浪微博用户的网络 “痕迹”, 较为可靠的获取南京成年人微博用户与本地人/外地人、熟人/陌 生人在网络社区中的交流差异。研究表明: (1)在网络社区中, 相较于外地人与陌生人, 受访者更倾向与本地人以及 熟人交流,网络社区中空间距离与关系距离的作用仍然存在; 2)受访者在网络社区中与本地人/外地人、熟人陌生 人的交流差异受社会经济属性、居住环境、微博使用频繁程度影响; 3 虽然理论上网络社区使得用户能够克服距离 阻力, 延伸其关系网络, 但空间距离与关系距离的存在使得实际生活中的社会关系网络仍然扎根于用户在网络社 区中的交流。
\end{abstract}

关 键 词: 空间距离; 关系距离; 网络社区; 信息通信技术; 新浪微博

\section{1 引言}

距离一直是地理学研究关注的核心概念与研 究议题(Tobler, 1970; 胡兆量, 1991; 王波等, 2013, 2014)。特别是, 随着以互联网为代表的信息通信 技术在全球的快速发展及在人们日常生活中的渗 透与影响,不断挑战着这一传统的规律, 部分学者 甚至发出“距离的死亡”“空间、城市与地理的终结” 等惊叹(O'brien, 1992; Cairncross, 2001)。而与此相 对应的是, 人流、物流、资本流仍然集聚在全球范围 内的重要节点城市, 并且城市间流的强度随着距离 的增加而逐渐衰减 (Goetz, 1992; Mitchelson et al, 1994; Matsumoto, 2004)。在信息时代下, 距离在实 体空间和虚拟空间中的二重作用, 不但没有削弱物 质要素在空间上的分散, 反而进一步强化空间的集
聚效应,特别是在高等级城市以及这些城市中心地 区的集聚(王波等, 2014)。但已有的研究大多聚焦 于距离在实体空间中活动的作用，而较少关注到距 离在虚拟空间中活动的作用。随着电话、短信、邮 件、QQ、微博等一系列电子交流方式的普及, “全球 本地化”(Glocalization)成为现代社会人们社会关系 的新特征,表现为: 在空间距离上不断延伸的社会 关系网络与基于本地的社会关系网络并存; 在关系 距离 ${ }^{\mathbb{1}}$ 上不断延伸的社会关系网络与基于熟人的社 会关系网络并存(Wellman et al, 1979)。以新浪微博 为代表的新型网络社区, 已经成为人们网络交流的 重要媒介之一。与面对面交流, 电话、短信、邮件等 传统交流方式不同, 新型网络社区的开放性特征为 人们提供了基于 “弱关系 ${ }^{2}$ ”的交流媒介,进一步挑 战传统的基于本地、熟人的社会关系网络。基于

收稿日期: 2016-03; 修订日期: 2016-06。

基金项目: 国家自然科学基金项目(41571146) [Foundation: National Natural Science Foundation of China, No.41571146]。

作者简介:王波(1987-),男,湖南衡阳人,博士生, 主要从事城市地理与区域规划研究,E-mail: wangbo_nick@163.com。

通讯作者: 甄峰(1973-), 男,陕西汉中人,教授,博导, 从事城市地理与区域规划、智慧城市研究, E-mail: zhenfeng@nju.edu.cn。

(1)关系距离指在社会关系中对方重要性的区别,例如熟人相对于陌生人关系距离更近。

(2)新型网络社区为人们提供了一个构建网络社会关系的媒介。从好友关系形成与联系的建立来看,这一网络社会关系并不

需要依托于生活中的社会关系。 of distance in online social networks: A case study of Sina micro-blog[J]. Progress in Geography, 35(8): 983-989.]. DOI: 10.18306/ dlkxjz.2016.08.007 
此, 本文以新浪微博为例, 探讨距离在网络社区中 的作用。

信息时代下不断普及的电子交流方式,引起了 学者对于电子交流与面对面交流间关系的关注。 有研究表明, 虚拟空间中的电子交流衍生于实体空 间中面对面交流的需求, 因此面对面交流的频次会 促进电子交流的频次(Tillema et al, 2008; 魏宗财 等, 2008)。但也有研究表明, 电子交流会替代面对 面交流, 用户在网络社区中花费的时间越多, 在实 体空间中与他人面对面交流的时间与频次越少 (Thulin et al, 2005; Veenhof et al, 2008)。同时, 相较 于以汽车、火车、飞机为代表的交通技术的进步, 信 息通信技术的进步更大程度降低了人们在网络社 区中跨越距离交流的时间与金钱成本, 引起了部分 地理学者对于距离在电子交流中作用的关注。例 如, Mok等(2010)基于 2005 年对 86 位美国北约克地 区居民的调查发现, 面对面交流主要集中在约 $8 \mathrm{~km}$ 的范围内, 电话交流主要集中在约 $160 \mathrm{~km}$ 的范围 内。与空间距离相比, 部分研究开始关注关系距离 的影响。对荷兰的研究表明, 空间距离在不同社会 关系下的作用表现不同(Tillema et al, 2008); 对瑞典 互联网年轻用户的研究发现, 用户倾向在网络中与 实际生活中的熟人交流(Thulin et al, 2005); 对美国 的研究也表明, 与非亲属相比, 人们倾向与亲属有 更频繁的电话和邮件联系(Mok et al, 2010)。

中国的传统东方文化向来重视社会关系(孙立 平, 1996; 陈爽英等, 2010)。改革开放前, 在乡村熟 人社会中, 人们倾向与村落范围内的人交流, 建立 起以生产队范围为基础的社会关系网络 (Fei, 1989); 在城市单位大院中, 人们倾向与单位院落内 的人交流, 建立起以单位大院为基础的社会关系网 络(Hazelzet et al, 2012)。随着改革开放后的快速城 市化进程, 一方面农村人口涌向城市, 来到城市工 作与生活; 另一方面城市内单位制改革, 单位院落 在城市空间中逐步瓦解(王桂新等, 2008)。因此, 有 必要进一步研究, 传统的基于小尺度(生产队村落、 单位大院)的本地、熟人的社会关系网络是如何影 响到人们在以 “弱关系”为基本特征的新型网络社 区中的交流。

在现有对电子交流方式的研究中, 几乎忽视了 对 Twitter、微博等新型网络社区的分析 (LibenNowell, 2005; 魏宗财等, 2008; Takhteyev et al, 2012; 甄峰等, 2012; 王波等, 2013)。当前的研究方
法主要分为: 问卷访谈与网络数据挖掘 2 类。其中, 网络数据挖掘是通过相关程序大规模获取网络用 户在 Twitter、微博等新型网络社区中遗留下的使用 痕迹(即“好友关系与交流记录”), 提供了相对更为 可靠的关系与交流频次数据, 从而使分析距离在网 络社区交流中的根植性规律成为可能 (LibenNowell, 2005)。但已有的分析大多是从关系的角度 出发,忽视了实际的交流情况(Liben-Nowell, 2005; Takhteyev et al, 2012; 王波等, 2013)。值得注意的 是, 网络社区中的好友关系并不代表用户间的实际 交流情况。同时,网络数据挖掘往往难以获取用户 可靠的社会经济属性,从而导致分析大多基于网络 数据的总体特征,而忽视了对用户社会经济属性等 要素的控制。这些要素不仅影响人们在实际生活 中的社会关系, 也可能影响人们在网络虚拟社区中 的交流。相对而言, 问卷访谈虽然可以得到可靠的 个人社会经济属性数据, 但交流频次与空间距离数 据却往往依赖于受访者的记忆与判断, 从而导致数 据的可靠性较低, 难以满足系统性研究距离在各种 电子交流方式的需求(Mok et al, 2010)。已有的问 卷调查一般包括: 对小规模样本 $(<100)$ 与其他人的 交流频次和空间距离的详尽调查(但交流频次仍然 取决于受访者的记忆), 以及对中等规模样本(500 700 左右)与其他人的交流频次和空间距离区间范 围的调查 (Thulin et al, 2005; Tillema et al, 2008; Mok et al, 2010)。本文尝试结合 2 种研究方法, 通 过问卷访谈获得受访者的社会经济属性数据, 同时 借助受访者在新浪微博中交流记录的核实, 获得他 们与本地人/外地人、熟人/陌生人交流差异的可靠 数据, 分析空间距离与关系距离的作用。需要指出 的是, 以本地人/外地人区分空间距离, 以熟人/陌生 人区分关系距离, 旨在探讨空间距离与社会距离在 基于 “弱关系”的新浪微博新型网络社区中的作用, 从而回应传统的本地熟人的社会关系网络是如何 反映在以 “弱关系”为基本特征的新型网络社区中 的交流。同时,也期待通过对问卷调查与网络数据 结合的尝试, 扩展已有的研究方法。

\section{2 研究方法}

在本文的问卷调查期间, 新浪微博拥有很高的 普及率, 全国共计有 2.7 亿微博用户 ${ }^{3}$ 。其中, 《新浪 微博元年白皮书》(2010)的调查数据显示, 新浪微博

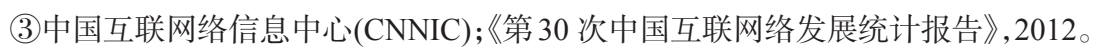


是国内市场知名度与用户使用率最高的主流微博 产品。因此, 本文选择新浪微博的研究具有一定的 代表性。

研究数据来自于 2012 年 9 月份在南京开展的 “网络信息时代居民行为调查问卷”。由于全城人 户调查的困难性, 本次调研选择在南京市 11 个区 (包括市区内的白下区、鼓楼区、建帮区、秦淮区、栖 霞区、下关区、玄武区、雨花区, 以及郊区的江宁区、 六合区、浦口区)的主要商业地区、大型公共场所以 及居住区随机发放了 1038 份问卷。问卷发放对象 为在南京工作与生活的成年居民; 除去无效问卷 后, 共得到 980 份有效问卷。问卷的调查内容包括: (1)居民的社会经济属性; (2)居住地与工作地情况; (3)信息通信技术的使用; (4)交通出行; (5)网络购物、 网络办公、网络社区等网络行为。本文的研究数据 来自问卷中的网络社区部分; 其中设置了针对新浪 微博用户的一系列问题, 共计 269 位访谈者完成该 部分问题, 作为本研究的样本。需要指出的是, 本 次样本并不能均衡代表整体南京的成年人微博用 户, 考虑到本文旨在分析本地/熟人的社会关系网络 是如何反映和作用于新浪微博中(而不是描述南京 成年人新浪微博用户的交流行为), 样本的一定偏 差并不会影响到对数据的多元变量分析(Babbie, 2007)。但需要注意的是, 本文的结论与讨论均是 针对本次调研中使用新浪微博并认真完成该部分 问卷的成年受访者。

在具体问题中, 受访者被要求查看近 2 周以来 他们在新浪微博中与他人交流的情况(包括回复、 转发、私信), 根据交流记录以及交流用户的个人属 性数据(注册地址是南京本地还是外地, 与受访者 是现实生活中的熟人还是陌生人), 分别统计或估 算与本地人/外地人、与熟人/陌生人的交流次数; 并 根据统计数据, 回答与本地人还是外地人交流次数 更多; 与熟人还是陌生人交流次数更多的问题。

\section{3 结果与分析}

首先是对样本统计资料进行描述性分析; 其次 在对相关变量介绍的基础上,采用二项逻辑回归分 析, 探讨受访者社会经济属性、居住地环境、信息通 信技术使用程度对网络社区交流中距离作用的 影响。

\section{1 样本变量及统计资料描述性分析}

基于调查的 269 位有效样本, 对空间距离与关 系距离在新浪微博中的作用予以描述 $($ 表 1$)$ 。总体 来看, $52.8 \%$ 的受访者与本地人交流更加频繁, $69.9 \%$ 的受访者与熟人交流更加频繁。

在对样本的统计中,包括社会经济属性、居住 地环境以及信息通信技术 3 个方面的变量。在社会 经济属性方面, 50.9\%的样本为男性; $65.4 \%$ 的样本 年龄在 30 岁以下; $63.2 \%$ 的样本拥有本科或本科以 上学历, 这与目前中国新浪微博在年轻人以及高教 育水平群体中的高使用率有关, 与发达国家情况类 似(Thulin et al, 2005); 90.3\% 的样本有工作, 并且 $80.3 \%$ 拥有南京户口。

表 1 被调查样本的基本情况统计表

Tab.1 Summary statistics of the variable values of the respondents

\begin{tabular}{|c|c|c|c|c|c|}
\hline 变量 & 赋值 & 样本量 & 比例/\% & 均值 & 标准差 \\
\hline \multirow[t]{2}{*}{ 性别 } & 男=1 & 137 & 50.9 & 0.51 & 0.501 \\
\hline & 女 $=0$ & 132 & 49.1 & & \\
\hline \multirow[t]{4}{*}{ 年龄 } & 18 19 岁, 赋值 1 & 43 & 16.0 & 2.26 & 0.814 \\
\hline & 21 29岁, 赋值 2 & 133 & 49.4 & & \\
\hline & 30 39 岁, 赋值3 & 73 & 27.1 & & \\
\hline & $\geqslant 40$ 岁,赋值 4 & 20 & 7.4 & & \\
\hline \multirow[t]{4}{*}{ 受教育水平 } & $\begin{array}{l}\text { 高中或高中 } \\
\text { 以下=1 }\end{array}$ & 24 & 8.9 & 2.70 & 0.835 \\
\hline & 大专院校=2 & 75 & 27.9 & & \\
\hline & 本科=3 & 129 & 48.0 & & \\
\hline & 硕士或以上=4 & 41 & 15.2 & & \\
\hline \multirow[t]{2}{*}{ 就业情况 } & 有工作=1 & 243 & 90.3 & 0.90 & 0.296 \\
\hline & 无工作 $=0$ & 26 & 9.7 & & \\
\hline \multirow[t]{2}{*}{ 南京户口 } & 有 $=1$ & 216 & 80.3 & 0.80 & 0.398 \\
\hline & 无 $=0$ & 53 & 19.7 & & \\
\hline \multirow[t]{2}{*}{ 居住社区类型 } & 单位社区=1 & 50 & 18.6 & 0.19 & 0.390 \\
\hline & 非单位社区 $=0$ & 219 & 81.4 & & \\
\hline \multirow[t]{2}{*}{ 居住地区位 } & 市区=1 & 181 & 67.3 & 0.67 & 0.470 \\
\hline & 郊区 $=0$ & 88 & 32.7 & & \\
\hline \multirow[t]{2}{*}{ 智能手机拥有 } & 有 $=1$ & 234 & 87.0 & 0.87 & 0.337 \\
\hline & 无 $=0$ & 35 & 13.0 & & \\
\hline \multirow{3}{*}{$\begin{array}{l}\text { 每天新浪微博 } \\
\text { 使用时间 }\end{array}$} & $<0.5 \mathrm{~h}$, 赋值 1 & 146 & 54.3 & 1.63 & 0.764 \\
\hline & $0.5 \sim 1.0 \mathrm{~h}$, 赋值 2 & 76 & 28.3 & & \\
\hline & $>1 \mathrm{~h}$, 赋值 3 & 47 & 17.5 & & \\
\hline \multirow{2}{*}{$\begin{array}{l}\text { 是否与本地人 } \\
\text { 交流更多 }\end{array}$} & 是 $=1$ & 142 & 52.8 & 0.53 & 0.500 \\
\hline & 否 $=0$ & 127 & 47.2 & & \\
\hline \multirow{2}{*}{$\begin{array}{l}\text { 是否与熟人交 } \\
\text { 流更多 }\end{array}$} & 是 $=1$ & 188 & 69.9 & 0.70 & 0.460 \\
\hline & 否 $=0$ & 81 & 30.1 & & \\
\hline
\end{tabular}


在居住地环境方面, $67.3 \%$ 的样本生活在市区， 这与城市的人口布局以及调研过程中调研地点的 选择有关。仅仅有 $18.6 \%$ 的样本生活在单位社区 ${ }^{(4}$ 中, 这反映了当前随着住宅商品化改革与建设, 单 位社区在城市空间结构中比例不断下降。

在信息通信技术使用方面, $87.0 \%$ 的样本拥有 智能手机, 这也与当前中国快速的移动信息化发展 有关, 特别是在年轻人群体中智能手机普及率不断 提升。 $54.3 \%$ 的样本每天使用新浪微博的时间在 $0.5 \mathrm{~h}$ 以内, $28.3 \%$ 和 $17.5 \%$ 的样本每天使用新浪微 博的时间分别达到 $0.5 \sim 1.0 \mathrm{~h}$ 和 $>1 \mathrm{~h}$ 。

\section{2 空间距离与社会距离的二项逻辑回归分析}

为分析距离在新浪微博社区交流中的作用, 分 别从空间距离与关系距离 2 个方面, 设立两组二分
虚拟因变量。其中,在空间距离方面,与本地人交 流更多 $=1$, 与外地人交流更多 $=0$; 在关系距离方面, 与熟人交流更多 $=1$, 与陌生人交流更多 $=0$ 。采用二 项逻辑回归模型进行回归, 其模型结构为:

$$
\ln \left(\frac{p}{1-p}\right)=\alpha+\sum_{i=1}^{n} \beta_{i} \chi_{i}
$$

式中: $p=P\left(y \mid \chi_{1}, \chi_{2}, \cdots, \chi_{n}\right)$ 为系列自变量 $\chi_{1}, \chi_{2}, \cdots, \chi_{n}$ 是 “与本地人交流更多”或 “与外地人交流更多”事 件(或: “与熟人交流更多”或“与陌生人交流更多” 事件)发生的概率; $\chi_{i}$ 为包括社会经济属性、居住地 环境以及信息通信技术 3 个方面的基本特征变量; $\alpha$ 表示常量; $\beta_{i}$ 为偏回归系数。回归分析的结果显 示(表 2), 基于空间距离与关系距离的模型整体检验 均十分显著。

表 2 新浪微博社区交流中距离作用的二项逻辑回归分析

Tab.2 Binary logistic regression of the role of spatial distance and relational distance in interpersonal interactions in Sina micro-blog

\begin{tabular}{|c|c|c|c|c|c|c|}
\hline & \multicolumn{3}{|c|}{ 模型 $1:$ 与南京本地人交流 } & \multicolumn{3}{|c|}{ 模型 $2:$ 与实际生活熟人交流 } \\
\hline & 系数 $(B)$ & 标准误差 & $\operatorname{Exp}(B)$ & 系数 $(B)$ & 标准误差 & $\operatorname{Exp}(B)$ \\
\hline 性别(参考指标:女性) & 1.262 & $0.348^{* * *}$ & 3.531 & 1.689 & $0.378^{* * *}$ & 5.413 \\
\hline \multicolumn{7}{|l|}{ 年龄(参考指标: 18 19岁) } \\
\hline 20 29岁 & 1.111 & $0.611^{*}$ & 3.038 & 2.585 & $0.645^{* * *}$ & 13.267 \\
\hline 30 39 岁 & 0.498 & 0.634 & 1.646 & 2.737 & $0.708^{* * *}$ & 15.438 \\
\hline$\geqslant 40$ 岁 & -1.063 & 0.772 & 0.345 & 1.927 & $0.821^{* *}$ & 6.868 \\
\hline \multicolumn{7}{|l|}{ 受教育水平(参考指标: 硕士及以上) } \\
\hline 高中或高中以下 & 0.929 & 0.786 & 2.532 & -0.298 & 0.763 & 0.742 \\
\hline 大专院校 & 1.269 & $0.543^{* *}$ & 3.599 & 0.063 & 0.596 & 1.065 \\
\hline 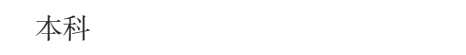 & 0.955 & $0.470^{* *}$ & 2.598 & 0.108 & 0.514 & 1.114 \\
\hline 就业情况(参考指标:无工作) & -1.368 & $0.818^{* * *}$ & 0.255 & 0.614 & 0.704 & 1.847 \\
\hline 南京户口(参考指标:无南京户口) & 3.862 & $0.652^{* * * *}$ & 47.58 & 0.777 & $0.449^{*}$ & 2.176 \\
\hline 居住社区类型(参考指标:非单位社区) & -0.047 & 0.445 & 0.954 & 0.913 & $0.532^{*}$ & 2.491 \\
\hline 居住地区位(参考指标:郊区) & 0.874 & $0.353^{* *}$ & 2.397 & -0.072 & 0.374 & 0.931 \\
\hline 智能手机拥有(参考指标: 无智能手机) & 0.019 & 0.546 & 1.019 & 0.137 & 0.638 & 1.147 \\
\hline \multicolumn{7}{|l|}{ 每天新浪微博使用时间 (参考指标: <0.5 h) } \\
\hline $0.5 \sim 1.0 \mathrm{~h}$ & -1.592 & $0.401^{* * *}$ & 0.203 & -2.431 & $0.486^{* * *}$ & 0.088 \\
\hline$>1.0 \mathrm{~h}$ & -1.854 & $0.492^{* * *}$ & 0.157 & -2.946 & $0.547^{* * *}$ & 0.053 \\
\hline 常数项 & -3.834 & $1.051^{* * *}$ & 0.022 & -1.903 & $1.010^{*}$ & 0.149 \\
\hline 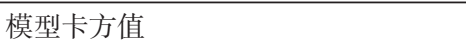 & \multicolumn{3}{|c|}{$138.401^{* * *}$} & \multicolumn{3}{|c|}{$119.332^{* * *}$} \\
\hline 拟合优度(Nagelkerke $R^{2}$ ) & \multicolumn{3}{|c|}{0.537} & \multicolumn{3}{|c|}{0.509} \\
\hline 解释度/\% & \multicolumn{3}{|c|}{80.7} & \multicolumn{3}{|c|}{82.9} \\
\hline 样本量 & \multicolumn{3}{|c|}{269} & \multicolumn{3}{|c|}{269} \\
\hline 自由度 & \multicolumn{3}{|c|}{14} & \multicolumn{3}{|c|}{14} \\
\hline
\end{tabular}

注:其中“*”、“**”和“***”分别表示 $0.1 、 0.05 、 0.01$ 的显著水平上显著(双尾检定)。

(4)本文中,单位社区既包括遗留下的单位大院的住宅区,也包括之后新建的有一定福利性质的单位分配房小区。 


\section{(1) 空间距离}

从空间距离来看,模型 1 的解释度为 $80.7 \%$ 。 模型主要影响因素包括性别、受教育水平、就业情 况、南京户口、居住地区位、每天使用新浪微博的时 间。与女性样本相比, 男性倾向于与南京本地人交 流的概率是女性的 3.531 倍, 表现出明显的性别差 异。以往的研究表明,女性往往在网络社区中参与 度更高(Tillema et al, 2008; 王波等, 2014)。本文进 一步发现, 女性更倾向于在这一开放社区中, 建立 与外地人的 “弱关系”网络。此外, 新浪微博使用时 间越长, 样本倾向与外地人交流的概率也越高。有 意思的是, 智能手机的拥有对与本地人或外地人的 交流倾向并无显著影响, 这可能与当前中国智能手 机的高拥有率有关, 也与智能手机在生活中扮演的 具体角色有关。例如有研究指出, 拥有一台智能手 机有时并不是追求智能手机的功能, 而是出于对身 份或 “面子” 的考量(Chu, 2008), 即: 拥有但不一定 使用或经常使用。

南京户口对与本地人交流的倾向有较明显的 影响, 拥有南京户口的样本倾向于与本地人交流的 概率是外地户口的 47.58 倍。考虑到生活在南京却 没有南京户口的样本多为在南京务工的农民工或 就学的大学生, 一方面他们在实际生活中与本地人 的关系网络较少, 另一方面可能也反映网络社区在 一定程度上发挥了维持他们与所属家乡或者“同为 异客”人们的社会关系的作用。

与未就业的样本相比, 已经就业的群体由于社 会关系更加广泛, 因而有更可能在网络社区中与外 地人交流。同理, 相对于拥有本科或大专学历的样 本, 拥有硕士学历的群体与外地人交流的可能性也 越高。

与 18 19 岁的样本相比, 20 29 岁的群体更倾 向与本地人交流。这可能与青少年群体对网络社 区更加开放的态度有关(Tillema et al, 2008)。在当 前的新浪微博社区中, 不少年轻人会就某个事件、 某位人、某个共同的兴趣爱好进行交流，而并不一 定关心对方与其在实际生活中的关系。这也反映 在模型 2 中对 “与实际生活中熟人交流更多” 的分析 结果中。与年轻人样本相比, 随着年龄的增长, 人 们更加倾向与熟人交流。

与生活在郊区的样本相比, 市区的样本倾向与 南京本地人交流。一方面, 市区的人口更加密集, 实际生活中, 在市区与本地人交流的机会更多从而
可能影响到人们的网络社区交流; 另一方面,与发 展较晚的郊区样本对比, 生活在市区的样本通常有 在南京更长的生活经历, 因而可能在网络社区交流 中更加关注本地人的用户与本地发生事件。

(2) 关系距离

从关系距离来看, 模型 2 的解释度为 $82.9 \%$ 。 模型主要影响因素包括性别、年龄、南京户口、居住 社区类型、与每天使用新浪微博的时间。与模型 1 类似,相对于女性样本, 男性倾向于与熟人交流的 概率是女性的 5.413 倍。每天使用新浪微博时间越 长, 倾向于与熟人交流的概率越低。

相对于拥有南京户口的样本, 没有南京户口的 群体倾向与熟人交流的概率低, 这可能与这部分群 体在南京熟人相对少有关, 这也呼应了模型 1 的结 论。同时,结合模型 1 中对他们与本地人交流倾向 的分析, 对于这部分群体, 网络社区在一定程度上 不仅维系着他们与家乡的社会关系纽带, 同时也帮 助他们在新环境下扩大社会关系网络、寻求更多社 会支持的作用,这与已有的大城市农民工的社会关 系研究一致(Liu et al, 2012)。从这点来看, 新型网 络社区给予这部分还没有“根”的群体一定的社会 资本价值,在未来社会管理中值得关注。

此外,生活在单位社区的样本更加倾向与熟人 交流。这也反映了在单位社区中的生活经历在网 络社区中的延续。在以往的生活中, 这部分群体习 惯与同在单位大院内(包括职工及其家人)共同生 活、居住的人们建立起基于熟人的社会关系,这一 习惯可能对他们在网络社区中的交流产生影响。

\section{4 结论与讨论}

本文通过对南京成年人在新浪微博中与本地 人/外地人、熟人/陌生人交流频率差异的分析,探讨 了空间距离与社会距离在网络社区中的作用。研 究发现, 现实生活中传统社会关系网络影响着人们 在新型网络社区中的社会关系网络,并体现在空间 距离与关系距离的作用中。特别是, 户口、单位社 区等要素显著影响了人们在网络社区中的交流行 为。同时,互联网的影响也不可忽视。新浪微博使 用时间越长,开放社区中的“弱关系”网络越脱离现 实社会中的传统社会关系网络, 表现为样本与外地 人、陌生人交流概率越大。本文的分析也呼应了对 科技、社会关系、地理距离三者关系的讨论 
(Licoppe, 2004)。随着网络应用的不断深人, 这一 科技力量给包括社会关系在内的日常生活带来更 大的影响, 从而进一步挑战传统的地理规律和概 念, 值得跟踪研究。

本文作为一个探索性的分析, 存在以下一些不 足: 首先, 由于本次研究来自于问卷中的一部分, 在 有限的时间里没有详尽调查样本在空间距离和关 系距离方面更细致的数据。在接下来更有针对性 的问卷设计中, 将对关系距离作进一步细分, 例如 按照亲缘关系划分为亲人与非亲人, 按照熟悉程度 进一步打分细分; 空间距离可以测量统计; 交流频 次则可统计面对面、电话、新型网络社区交流的具 体频次。这类详实数据将有助于更深人地分析用 户在虚拟空间中的交流轨迹与距离衰减效应, 这有 待于在后续调研分析中补充改进。其次, 家庭结 构、社会关系网络、人际交流主观态度等这些可能 影响电子交流选择与方式的变量, 在本次研究中还 没有被涵盖。最后, 本文也是在传统问卷调研中引 人网络数据的一个尝试, 在网络大数据背景下, 这 一尝试还有待在后续研究中进一步改进和深化, 使 不同类型数据更好服务于相关研究。

\section{参考文献(References)}

陈爽英, 井润田, 龙小宁, 等. 2010. 民营企业家社会关系资 本对研发投资决策影响的实证研究 [J]. 管理世界, (1): 88-97. [Chen S Y, Jin R T, Long X N, et al. 2010. Minying qiyejia shehui guanxi ziben dui yanfa touzi juece yingxiang de shizheng yanjiu[J]. Management World, (1): 8897.]

胡兆量. 1991. 地理学的基本规律 [J]. 人文地理, 6(1): 9-13.

[Hu Z L. 1991. The basic rules of geography[J]. Human Geography, 6(1): 9-13.]

孙立平. 1996. “关系”、社会关系与社会结构 [J]. 社会学研 究, (5): 22-32. [Sun L P. 1996. "Guanxi", shehui guanxi yu shehui jiegou[J]. Sociological Studies, (5): 22-32.]

王波, 甄峰, 魏宗财. 2014. 南京市区活动空间总体特征研 究: 基于大数据的实证分析 [J]. 人文地理, 29(3): 14-21, 55. [Wang B, Zhen F, Wei Z C. 2014. The research on characteristics of urban activity space in Nanjing: An empirical analysis based on big data[J]. Human Geography, 29(3): 14-21, 55.]

王波, 甄峰, 席广亮, 等. 2013. 基于微博用户关系的网络信 息地理研究: 以新浪微博为例 [J]. 地理研究, 32(2): 380391. [Wang B, Zhen F, Xi G L, et al. 2013. A study of cybergeography based on micro- blog users' relationship:
With a case of Sina micro-blog[J]. Geographical Research, 32(2): 380-391.]

王桂新, 沈建法, 刘建波. 2008. 中国城市农民工市民化研 究: 以上海为例 $[\mathrm{J}$ ]. 人口与发展, 14(1): 3-23. [Wang G X, Shen J F, Liu J B. 2008. Citizenization of peasant migrants during urbanization in China: A case study of Shanghai[J]. Population \& Development, 14(1): 3-23.]

魏宗财, 甄峰, 张年国, 等. 2008. 信息化影响下经济发达地 区个人联系网络演变: 以苏锡常地区为例 $[\mathrm{J}]$. 地理科学 进展, 27(4): 82-88. [Wei Z C, Zhen F, Zhang N G, et al. 2008. The evolution characteristics of personal communication network of economically developed regions under the influence of infomationization: A case study of SuzhouWuxi- Changzhou area[J]. Progress in Geography, 27(4): 82-88.]

甄峰, 王波, 陈映雪. 2012. 基于网络社会空间的中国城市网 络特征: 以新浪微博为例 [J]. 地理学报, 67(8): 10311043. [Zhen F, Wang B, Chen Y X. 2012. China's city network characteristics based on social netwrok space: An empirical analysis of Sina micro-blog[J]. Acta Geographica Sinica, 67(8): 1031-1043.]

Cairncross F. 2001. The death of distance: How the communications revolution is changing our lives[M]. Boston, MA: Harvard Business Press.

Chu W C R. 2008. The dynamics of cyber China: The characteristics of Chinese ICT use[J]. Knowledge, Technology \& Policy, 21(1): 29-35.

Davies H, Leung T K P, Luk S T K, et al. 1995. The benefits of "Guanxi": The value of relationships in developing the Chinese market[J]. Industrial Marketing Management, 24 (3): 207-214.

Fei X T. 1989. Rural development in China: Prospect and retrospect[M]. Chicago, IL: University of Chicago Press.

Goetz A R. 1992. Air passenger transportation and growth in the U.S. urban system, 1950-1987[J]. Growth and Change, 23(2): 217-238.

Hazelzet A, Wissink B. 2012. Neighborhoods, social networks, and trust in post-reform China: The case of Guangzhou[J]. Urban Geography, 33(2): 204-220.

Liben-Nowell D, Novak J, Kumar R, et al. 2005. Geographic routing in social networks[J]. Proceedings of the National Academy of Sciences of the United States of America, 102 (33): 11623-11628.

Licoppe C. 2004. 'Connected' presence: The emergence of a new repertoire for managing social relationships in a changing communication technoscape[J]. Environment and Planning D: Society and Space, 22(1): 135-156. 
Liu Y, Li Z G, Breitung W. 2012. The social networks of newgeneration migrants in China's urbanized villages: A case study of Guangzhou[J]. Habitat International, 36(1): 192200.

Matsumoto H. 2004. International urban systems and air passenger and cargo flows: Some calculations[J]. Journal of Air Transport Management, 10(4): 239-247.

Mitchelson R L, Wheeler J O. 1994. The flow of information in a global economy: The role of the American urban system in 1990[J]. Annals of the Association of American Geographers, 84(1): 87-107.

Mok D, Wellman B, Carrasco J. 2010. Does distance matter in the age of the Internet[J]. Urban Studies, 47(13): 27472783.

O'brien R. 1992. Global financial integration: The end of geography[M]. New York: Council on Foreign Relationship Press.

Takhteyev Y, Gruzd A, Wellman B. 2012. Geography of twit- ter networks[J]. Social Networks, 34(1): 73-81.

Thulin E, Vilhelmson B. 2005. Virtual mobility of urban youth: ICT-based communication in Sweden[J]. Tijdschrift voor Economische en Sociale Geografie, 96(5): 477-487.

Tillema T, Dijst M J, Schwanen T. 2008. Electronic and faceto-face communication in maintaining social relationships $[\mathrm{C}] / /$ Colloquium Vervoersplanologisch Speurwerk. Utrecht, Netherlands: Utrecht University.

Tobler W R. 1970. A computer movie simulating urban growth in the Detroit region[J]. Economic Geography, 46: 234240.

Veenhof B, Wellman B, Quell C, et al. 2008. Isolation, cohesion or transformation? How Canadians' use of the Internet is shaping society[R]. Ottawa, Canada: Statistics Canada.

Wellman B, Leighton B. 1979. Networks, neighborhoods, and communities approaches to the study of the community question[J]. Urban Affairs Review, 14(3): 363-390.

\title{
The role of distance in online social networks: A case study of Sina micro-blog
}

\author{
WANG Bo ${ }^{1}$, ZHEN Feng ${ }^{2,3 *}$ \\ (1. Department of Geography, The University of Hong Kong, Hong Kong 999077, China; \\ 2. School of Architecture and Urban Planning, Nanjing University, Nanjing 210093, China; \\ 3. Human Geography Research Center of Nanjing University, Nanjing 210093, China)
}

\begin{abstract}
The rapid development of information and communication technologies has brought numerous electronic communication modes ((mobile) phone, short message service, email, and recently the popular online social networking sites) that have been expected to influence the role of spatial and relational distances in interpersonal interactions. Based on online "traces" of Sina micro-blog users' interactions in the last two weeks, respondents in our questionnaire survey were required to record and answer questions of (1) who did you interact more often, locals or non-locals and (2) who did you interact more often, acquaintances or strangers in real life. Answers were used in our analysis of the role of spatial distance and relational distance in online social networks. Our findings show that (1) there is a clear tendency of respondents interacting with locals and acquaintances in their real life; (2) respondents' tendency to interact with locals and acquaintances is impacted by their socioeconomic traits, living environment, and information and communication technologies use; (3) theoretically online social networking sites enable users to overcome the distance decay effects and thereby to enlarge their social networks. However, spatial and relational distances sustained the social networks in real life in interpersonal interactions in online social networks.
\end{abstract}

Key words: spatial distance; relational distance; online social network; information and communication technology; Sina micro-blog 